\title{
THE EFFECT OF PRE-LACTEAL FEEDING ON FULL BREASTFEEDING IN NAIROBI, KENYA
}

\author{
Lakati $\mathrm{AS}^{1}$, Makokha $\mathrm{OA}^{1}$, Binns $\mathrm{CW}^{2}$ and Kombe $\mathrm{Y}^{3}$
}

\begin{abstract}
Objective: To assess the effect of pre-lacteal feeding on full breastfeeding in the first six months of life in selected hospitals, Nairobi Province. Pre-lacteal feeding has been internationally discouraged because of its negative effect on the duration of breastfeeding.

Methods: A prospective cohort design was used with a sample of 692 mother-infant pairs recruited at birth and followed up until 6 months of age. The sample was drawn from five major hospitals in Nairobi. Data was analyzed using the SPSS computer software. Descriptive analysis was used on all variables. Chi-test was used for univariate analysis. Logistic regression analysis was used to determine the effect of pre-lacteal feeding on full breastfeeding.

Results: Slightly more than half $(\mathbf{5 8 . 8 \%})$ of the mothers were formally employed and their mean age was $\mathbf{2 8 . 3}$ \pm 4.9 years. The mean income was KES $26,360 \pm 34,696$. The mean birth weight of the infants was $3.24 \pm 0.43 \mathrm{~kg}$ and $53.3 \%$ were males. The prevalence of pre-lacteal feeding was $26.8 \%$ (95\% CI $23.5 \%-30.1 \%$ ). The most common pre-lacteal feeds used at all hospitals were infant formula and glucose solution. There were significant $(\mathbf{P}<0.05)$ differences across the five study hospitals in this practice. In one hospital nearly all $(93 \%)$ infants received a pre-lacteal feed. The use of pre-lacteal feeding was significant predictor for early cessation of full breastfeeding at 6, 10, 14 and 19 weeks.

Conclusions: The practice of giving pre-lacteal feeds is a key determinant of early cessation of full breastfeeding. Some hospitals do not appear to be adhering to international recommendations on infant feeding. Polices to promote exclusive and full breastfeeding are necessary to enable infants to attain optimal health and lead to achievement of the Millennium Development Goals in our settings.
\end{abstract}

\section{Introduction}

Breastfeeding offers short term and long health benefits to mothers and children $(1,2)$. Because of this evidence, research on breastfeeding has focused on factors that affect attainment of the optimal duration of breastfeeding. The World Health Organization (WHO) strategy for infant feeding strongly discourages the use of pre-lacteal feeding (3). Pre-lacteal feeds are defined as "any feeds given before the onset of lactogenesis II, which is the onset of copious lactation that occurs within four days of birth"(4).

The vast majority of mothers can and should breastfed, just as the vast majority of infants can and should be breastfed. Only under exceptional circumstances can mothers milk be considered unsuitable for her infant (3, 5-7). Supplements such as water, glucose water, formula and other fluids should not be given to breastfed new born infants $(5,8)$. The World Health Organization (WHO) and United Nations Children's Fund (UNICEF) in 1991(9) launched the BabyFriendly Hospital Initiative to increase breastfeeding rates worldwide. Hospitals are expected to implement the ten steps to successful breastfeeding. Step number 6 states that new born infants should be fed on breastmilk only unless medically indicated and under no circumstances should breast milk

Correspondence to: Alice Lakati: Lakati@yahoo.com : Cell phone: 254 - 722840 012. P.O BOX 60720-00200 Nairobi

${ }^{1}$ Institute of Tropical Medicine and Infectious Diseases (ITROMID)Jomo Kenyatta University-Kenya ${ }^{3}$ Kenya Medical Research InstituteKEMRI-Kenya, ${ }^{2}$ School of Public Health Curtin University of Technology Western Australia GPO Box U1987 Perth WA substitutes, feeding bottles or pacifiers be given to new born infants.

The use of pre lacteal feeding has remained high despite the international recommendation that discourage the use. In Singapore $18 \%$ of infants are reported to have pre-lacteal feeds(10), while in India its as high as 93\%(11) or $74 \%(12)$ in rural communities. In The Gambia, the use of pre lacteal feeds was reported to be common with initiation of breastfeeding being delayed beyond one day. Infants were given either water, milk and water, breast milk from a wet nurse among other liquids (13).

The effect of pre lacteal feeds on breastfeeding has been reported especially in developed and developing countries. In La Paz Boliva (14), the use of pre lacteal feeds was significantly $(\mathrm{P}<0.0001)$ associated with shorter duration of exclusive breastfeeding. The prelacteal feeds given included, infant formula, tea, honey, salt and others types of drinks. A study in Xinjiang, People's Republic of China, found that the use of pre lacteal feeds such as water and formula had a negative impact on the initiation of breastfeeding (15). An earlier study in Central America had shown the association between use of pre-lacteal food and late initiation of breastfeeding. Perez-Escamilla et al (1996)(16) found that, milk water based prelacteal feeds among other variables were inversely associated with exclusive breastfeeding among 0-6 months old infants. Despite previous studies reporting the effect of pre-lacteal feeding on exclusive and general breastfeeding duration, health workers continue to play a major role in promoting the practice. In Nigeria, (17) some doctors and nurses were found to routinely prescribed pre lacteal feeds 
for mothers who were considered to have delayed lactation. The pre lacteal feeds used were infant formula and glucose drinks, and $35 \%$ were found to routinely advise giving prelacteal feeds within the first one hour after delivery. In Kenya the guidelines for infant and young child feeding discourage the use of prelacteal feeding (8). The main objective of this study was to determine the use and effect of prelacteal feeding in five major maternity hospitals in Nairobi.

\section{Methods}

A prospective cohort design was used. Cohorts comprised of mother-infant pairs recruited at birth and follow up until they were six months of age. Maternity hospitals offering maternity services in Nairobi the capital city of Kenya was the study area. Five maternity hospitals were purposively selected based on the number of deliveries per day and availability of maternal and child health clinics $(\mathrm{MCH})$. The $\mathrm{MCH}$ clinics were used to follow up motherinfant pairs in the first six months. A sample of 692 mother-infant pairs was recruited between November-2007 to March 2008. Recruitment was carried out at the maternity wards of the study hospitals before the mothers were discharged.

The inclusion criteria were mothers who reside in Nairobi, full term babies weighing $2 \mathrm{~kg}$ and above and mothers who were medically fit to participate in the study as advised by the nurses. Data was collected using a structured questionnaire. The questionnaire collected information on background information, delivery variables, and hospital practices. A follow up questionnaire was used monthly to obtain information on infant feeding practices.

All data was coded, entered and analyzed using the SPSS (Statistical Package for Social Sciences) version 18.0. Descriptive statistics involved measures of central tendency and dispersion for all continuous variables and frequencies and proportions for categorical variables. Chi-square tests were used at univariate level to determine variables associated with full breastfeeding.

Multivariate logistic regression analysis was employed to determine which individual variables could best predict early introduction to complementary feeding. All variables found to be significantly $(\mathrm{P}<0.05)$ associated with complementary feeding were included in the full model. The full model was reduced using the backward stepwise procedure and the fitness of the model was assessed at every step to avoid dropping non-significant variables that affected the model fitness.
The breastfeeding definitions used in this study are;

Exclusive breastfeeding (18): breastfeeding while giving no other food or liquid, not even water with an exception of western medicine since birth. Full breastfeeding: breastfeeding (19); infants receive breast milk and fluids such as water or other ritualistic feeds, that may be given infrequently. In this study the main cause of the shift from exclusive breastfeeding to full breastfeeding was the mother's practices of giving sips of water to infants occasionally. Therefore this study determined the effect of pre-lacteal feeding on full breastfeeding.

Complementary feeding (18): giving infants other foods in addition to breast milk. The additional foods can be liquids or solids. The early introduction to complementary feeding before six months was leading to cessation of full breastfeeding.

Ethical approval was granted by the Kenya Medical Research Institute (KEMRI) ethical review committee and the participating hospitals ethics committees. The names of the participating hospital are not indicated for confidentiality purposes.

\section{Results}

More than one third (38.6\%) of the mothers were recruited from the hospital labeled one and $23.7 \%$ were from hospital labeled two (Table 1) and only 14 mothers could be recruited from hospital five because of the low number of deliveries at the time of the study. Majority $(87.1 \%)$ of the mothers in the study were married, $10.6 \%$ single and the rest were either separated or divorced. Slightly above half $(56.5 \%)$ of the mothers had college and university level of education. The average age was $28.3 \pm 4.9$ years $(95 \%$ CI $27.97-28.70)$. The youngest mother was 17 years and the oldest was 45 years.

Nearly all $(99.1 \%)$ of the mothers reported to have attended antenatal clinics (ANC) during pregnancy, only one mother reported not to have attended ANC services during pregnancy. Slightly below half $(43.8 \%)$ of the mothers were having their first babies and a few (4.9\%) were having the fourth baby and above.

Slightly above half $(56.5 \%)$ of the mothers had a normal vaginal delivery and a third $(33.1 \%)$ had caesarean delivery. Majority $(72.8 \%)$ reported not to have had any complications during delivery. Less than a third (27.2\%) reported to have had complications such as prolonged labour, high blood pressure, foetal distress among others. These mothers however, were well at the time of the interview and they all had healthy babies. 
The use of pre-lacteal feeding was common. Almost a third $(26.8 \%)$ of the infants were found to have had a pre-lacteal feed as the first feed before breastmilk. Infant formula was the most common $(85.2 \%)$ pre-lacteal feed used, followed by glucose solution $(12.1 \%)$.

The use of pre-lacteal feeds differed significantly $\quad\left(\chi^{2}=278.634 ; \quad 4 d f ; \quad \mathrm{P}<0.001\right)$ between the five hospitals. Nearly all 92, $(93.9 \%)$ of the infants from hospital labeled four had received a pre-lacteal feed and $71.4 \%$ (10) at hospital five. The mother's highest level of education was significantly associated with prelacteal feeding. As the level of education of the mother increases the proportion of infants receiving pre-lacteal feeds increased. Among infants whose mothers who had primary level of education, $14.9 \%$ had pre-lacteal feeds as compared with $30.1 \%$ and $48.5 \%$ of infants whose mothers had college and university educational respectively. Pre-lacteal feeding also differed significantly $(\mathrm{P}<0.001)$ with mode of delivery. Infants born through caesarean section were more likely $(47.4 \%)$ to receive pre-lacteal feeds compared to vaginal delivery $(19.3 \%)$. Marital status and birth order were however, not associated with pre-lacteal feeding. Table 1 presents the distribution of the background characteristics of the study population and whether pre-lacteal feed was given.

The use of pre-lacteal feeding at birth was found to significantly $(\mathrm{P}<0.05)$ affect the prevalence of full breastfeeding using univariate analysis. The Chi-square test with Yates correction was used. Infants who had pre-lacteal feed had lower rates of full breastfeeding at 6 , 10, 14 and 19 weeks compared to infants who did not received a pre-lacteal feed. At 19 weeks only $14.3 \%$ of these infants were fully breastfeeding as compared to $33.7 \%$ of those who had not received a complementary feed. By 23 weeks the effect of pre-lacteal feeding was no longer significant (Table 2)

Multivariate logistic regression analysis was used to determine significant predictors for cessation of full breastfeeding. Variables included in the model were hospital of recruitment, highest level of education, age group and use of pre-lacteal feeding. After controlling for other predictors for early cessation of full breastfeeding, the use of prelacteal feeding remained as a significant predictor for early introduction of complementary feeding hence cessation of full breastfeeding at $6,10,14$ and 19 weeks. Infants who had a pre-lacteal feeds had higher odds for early complementary feeding than infants who had breastmilk as a first feed. Hospital four was a significant predictor for early cessation of breastfeeding at 6 weeks $0 \mathrm{R}=10.91$. This variable was related to pre-lacteal feeding and was therefore removed from the model (Table 3)

\section{Discussion}

The use of pre-lacteal feeds as the first food was very common in this study. Just over one quarter $(26.8 \%)$ of the infants had received prelacteal feeds. This prevalence is lower than what was reported in Bangladesh at community level of $93 \%(11)$. However, it higher than $18 \%$ reported in Singapore at hospital level (10). The use of pre-lacteal feeds has been reported to higher at community level in Bangladesh especially in infants born at home(11). In The Gambia, the use of pre lacteal feeds was reported to be common with initiation of breastfeeding being delayed beyond one day. Infants were given either water, milk and water, breast milk from a wet nurse among other liquids (13). In Jaipur City, India, the use of prelacteal feeds given by mothers was found to be very high at $96 \%$. The pre-lacteal feeds given included plain water, jaggery with ghee/oil, sugar water and other local liquids and oils(20).

In this study the type of pre lacteal feed used in this study was mainly infant formula or glucose solution and water. This practice was significantly $(\chi 2=278.63 ; 4 \mathrm{df} ; \mathrm{P}<0.001)$ different across the study hospitals with nearly all $(93.9 \%)$ of the infants from hospital four receiving a pre-lacteal feed as the first feed. At univariate level the use of pre-lacteal feeds was significantly $(\mathrm{P}<0.05)$ associated with early introduction of complementary feeding at 6 weeks, 10 weeks, 14 weeks and 19 weeks. Prelacteal feeding was also found a significant $(\mathrm{P}<0.05)$ predictor for early introduction of complementary feeding at multivariate level. These finding shows some hospitals and health workers contribute greatly to this practice that is discouraged by the Baby Friendly initiative.

The effect on pre lacteal feeds has also been reported in La Paz Boliva (14) among 518 infants. The use of pre lacteal feeds was significantly $(\mathrm{P}<0.0001)$ associated with shorter duration of exclusive breastfeeding. A study in Xinjiang, People's Republic of China, found that the use of pre lacteal feeds such as water and formula had a negative impact on the initiation of breastfeeding (15).

Previous studies had shown the association between use of pre-lacteal food and late initiation of breastfeeding. Perez-Escamilla et al (16) found that, milk water based pre- lacteal feeds among other variables were inversely associated with exclusive breastfeeding among 0-6 months old infants. Despite previous studies reporting the effect of pre-lacteal feeding on exclusive and general breastfeeding duration, health workers continue to play a major role in 
promoting the practice. A study of doctors and nurses in Nigeria (17), found that some doctors and nurses routinely prescribed pre lacteal feeds for mothers who were considered to have delayed lactation. The pre lacteal feeds prescribed were infant formula, glucose drinks, $35 \%$ were found to usually advise giving prelacteal feeds within the first one hour after delivery. The use of pre lacteal feeds in this study indicates that health workers played a role in this practice as all pre lacteal feeds were given by health workers in the respective study hospitals.

Clinician's options and practices are associated with continuation of exclusive breastfeeding. A study in the US (21) found that clinicians practices regarding formula supplementation of healthy infants and their opinions about the importance of breastfeeding were associated with the likelihood that mothers will continue exclusive breastfeeding. This US study recommended that: polices to enhance clinicians abilities to address breastfeeding problems within the constraint of their busy practices could improve their ability to support exclusive breastfeeding. The early cessation of exclusive and full breastfeeding in our study was significantly associated $(\mathrm{P}<0.05)$ with the use of pre-lacteal feeds. Health workers and hospital practices can hinder or promote attainment of optimal infant feeding.

In Kenya the guidelines on infant and young child feeding indicate that breastfeeding is the safest method of feeding for the first six month and no other food or drink (no pre lacteal) should be given as breast milk contains all the nutrients that a baby need for the first six months of life (8). Despite these guidelines it is evident from this study that health workers and hospitals, need to be trained to appreciate and implement this recommendation. It was also observed that each hospital had its specific ways of providing services at the maternity wards and ANC clinics. At the hospital four mothers were given written guidelines on infant feeding which contradict WHO recommendation of exclusive breastfeeding. Hospital three on the other hand encouraged exclusive breastfeeding and this could have contributed to the significant differences in the duration of full breastfeeding.

ANC services are important in providing education on breastfeeding and complementary feeding. In this study nearly all $(99.1 \%)$ of the mothers had attended ANC clinics during pregnancy. Only one mother from hospital one reported to have never utilized ANC services during pregnancy. There however significant $(\mathrm{P}<0.01)$ differences in between receiving education on infant feeding during pregnancy. Hospital two had less than half $(42.7 \%)$ of mothers reporting to have either received information. These findings indicate that not all ANC clinics provide education on infant feeding as recommended by the Kenya infant feeding guidelines (8).

Support from health workers was found improve outcomes in uptake of breastfeeding in prospective randomized open trial in the US (22). Mothers in the intervention were more likely to report exclusive breastfeeding at 4 weeks. This evidence can also be compared to the support mothers from the hospital three received and contributed to longer duration of exclusive and full breastfeeding in this group of mothers.

There are several limitations that need to be considered when interpretation the results of this study. The sample was restricted to the five major hospitals in Nairobi Province. While the hospitals selected were representative of the province, this should be born in mind as other urban town in Kenya may have differences with our study hospital. As socio-economic changes continue to occur in Nairobi, it will be important to repeat cohort studies so that breastfeeding is promoted in Kenya urban towns. Some study subjects were lost during follow up. This resulted from the January 2008 election crisis in Nairobi whereby some mothers changed their clinics and could not be traced. The sample that completed the study was found adequate to detect significant differences. Mothers who were lost to follow up were not significantly $(\mathrm{P}>0.05)$ different in their background characteristics with the ones who completed the study.

\section{Conclusions and Recommendations}

The hospital practice of giving pre-lacteal feeds is a key determinant of early cessation of full breastfeeding. Infants who had pre lacteal feeds as their first feed had significantly shorter duration of full breastfeeding than infants who had breast milk as a first feed. The use of prelacteal feeds differed significantly across different maternity hospitals with some hospitals having high rates of the use of pre-lacteal feeds. This practice has been discouraged by WHO and UNICEF and Kenya Ministry of Health/Public Health infant feeding guidelines.

Programs for continued medical education for health workers are necessary in all hospitals offering maternity services. This is to further update health workers on the WHO/UNICEF guidelines on infant feeding. This will contribute to the correct timing of complementary feeding which is an important indicator in the Millennium Development Goals. This will therefore lead to achievement of the MDG'S in our settings. 


\section{Acknowledgments}

The authors would like to acknowledge the support from the maternity staff of the five participating hospitals, the mothers who participated and the research assistants who assisted the principal author in data collection for six months. The study was partially funded supported by the Directorate of Personnel Management-Kenya

\section{Competing interest}

The authors declare that they have no competing interest

Table 1: Background characteristics of the study population and whether pre-lacteal feed was given

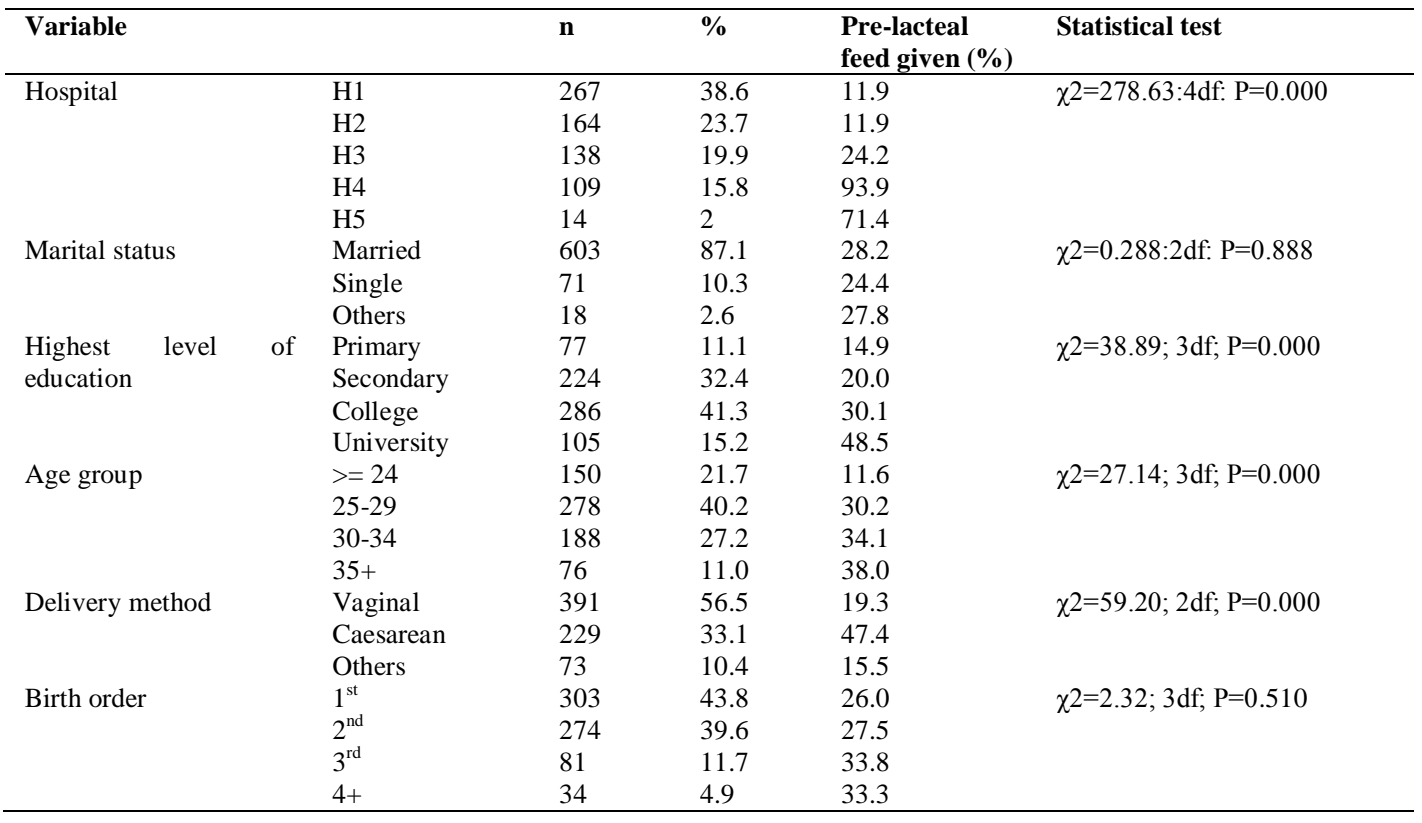

Table 2: Effect of pre-lacteal feeding on full breastfeeding at different weeks of follow up: Univariate analysis

\begin{tabular}{|c|c|c|c|c|c|}
\hline \multirow{2}{*}{ independent variables } & \multicolumn{5}{|c|}{ \% of infants on full breastfeeding at various times of follow up } \\
\hline & 6 weeks $(n=501)$ & 10 weeks $(n=342)$ & 14 weeks $(n=327)$ & 19 weeks (327) & 23 weeks (262) \\
\hline \multicolumn{6}{|c|}{ baby had pre-lacteal feed as first feed } \\
\hline yes & $74.6 \%$ & $61.1 \%$ & $38.1 \%$ & $14.3 \%$ & $13.9 \%$ \\
\hline no & $87.3 \%$ & $76.0 \%$ & $55.1 \%$ & $33.7 \%$ & $23.1 \%$ \\
\hline statistical test $* *$ & $\begin{array}{l}\chi 2 \text { yates }=10.59: \quad 1 \mathrm{df} \\
\mathrm{p}<0.05(0.001)\end{array}$ & $\begin{array}{l}\chi 2 \text { yates }=7.11: 1 \mathrm{df} \\
\mathrm{p}<0.05(0.008)-1\end{array}$ & $\begin{array}{l}\chi 2 \text { yates }=7.39: 1 \mathrm{df} \\
\mathrm{p}<0.05(0.006)-2\end{array}$ & $\begin{array}{l}\chi 2=64.57: \quad 1 \mathrm{df} ; \\
\mathrm{p}<0.01(0.000)-3\end{array}$ & $\begin{array}{l}\chi 2 \text { yates }=1.12: 1 \mathrm{df} ; \\
\mathrm{p}>0.01(0.132)\end{array}$ \\
\hline
\end{tabular}

Table 3: Effect of pre-lacteal feeding on full breastfeeding at different weeks of follow up: Logistic regression analysis

\begin{tabular}{|l|l|l|l|l|}
\hline $\begin{array}{l}\text { Week of } \\
\text { follow } \\
\text { up }\end{array}$ & $\begin{array}{l}\text { Wald } \\
\text { statistics }\end{array}$ & P value & $\operatorname{Exp(B)}$ & $\begin{array}{l}\text { Odds } \\
\text { ratio }\end{array}$ \\
\hline 6 weeks & 14.852 & 0.000 & 4.031 & 56.3 \\
\hline $\begin{array}{l}10 \\
\text { weeks }\end{array}$ & 4.311 & 0.038 & 1.873 & 6.57 \\
\hline $\begin{array}{l}14 \\
\text { weeks }\end{array}$ & 4.868 & 0.027 & 1.868 & 6.47 \\
\hline $\begin{array}{l}19 \\
\text { weeks }\end{array}$ & 4.598 & 0.032 & 2.287 & 9.84 \\
\hline
\end{tabular}

\section{References}

1. 1. Kramer MS, Guo T, Platt WR, Shapiro R, Collet J-P, Chalmers B, et al. Breastfeeding and Infant Growth: Biology or Bias? Pediatrics. 2002;110(10):343-7.

2. 2. Horta B, Bahl R, Martines J, Victoria C. Evidence on Long-term effects of Breastfeeding: Systematic Review and Meta-analyses. Geneva: WHO; 2007.

3. 3. WHO. Global Strategy for Infant and Young Child Feeding. Geneva: World Health Organization; 2003.

4. 4. Neville M, Morton J. Physiology and endocrine changes underlying human lactogenesis 11. Journal of Nutrition 2001;131:3005-8.

5. 5. AAP. Breastfeeding and the Use f Human Milk. 5. AAP. Breastfeeding and

6. Butte NF, Lopez-Alarcon MG, Garza C. Nutrient Adequacy of Exclusive Breastfeeding for the term infant during the first six months of life. World Health Organization Bulletin. 2002.

7. 7. WHO. Acceptable Medical Reasons for use of Breastmilk Substitutes. Geneva: World Health Organization; 2009.

8. 8. GOK, UNICEF, WHO. Infant \& Young Child Feeding in the Context of HIV/AIDS. Nairobi: Ministry of Health Kenya; 2003.

9. 9. WHO Ua. Baby-Friendly Hospital Intiative; Revised, Updated and Expanded for Integrated Cae. Geneva: World Health Organization United Nations Children Education Fund; 2006. 
10. 10. Koosha A, Hashemifesharaki R, Mousavinasab $\mathbf{N}$. Breastfeeding patterns and factors determining exclusive Breastfeeding patterns and factors determining exclusive
breastfeeding. Singapore Medical Journal. 2008;49(12): 1002-06.

11. 11. Tarannum S, Hyder S. Pre-lacteal feeding practices in a rural area of Bangladesh. Bangladesh: BRAC-ICDDR,B Joint Research Project Dhaka; 1998.

12. 12. CORT. Breastfeeding practices in two blocks of east Singbhum district Jharkhand. Gujarat-India: Centre for Operations Research and Training (CORT); 2002.

13. 13. Semega-Janneh I, Bohler E, Holm H, Matheson I, G H-O. Promoting breastfeeding in rural Gambia: combining traditional and modern knowledge. Health policy and planning. 2001;16(2):199-205.

14. 14. Ludvigsson J. Breastfeeding intentions, patterns, and determinats in infants visiting hospitals in La Paz Bolivia. BMC Pedatrics. 2003;3(5)

15. 15. Xu F, Binns $C$, Theng $S$, Wang $Y$, Zhao $Y$, Lee $A$. 15. Xu F, Binants of excuive breastfeeding duration in Xinjiang, PR China. Asia-Pacific Journal of Clinical Nutrition 2007;16(2):316-21.

16. 16. Perez-Escamilla R, Segura-Millan S, Canahuati J, Allen $H$. Prelacteal feeds are negatively associated with breastfeeding outcome in Honduras. Community and International Nutrition. 1996:2765-73.

17. 17. Joel-Medewase V, Fadero F, Oyedeji G. Pre-Lacteal feeding practices of doctors and nurses in a state and teaching hospital. The internet journal of nutrition and wellness. 2007;3(1).

18. 18. WHO. Indicators for Assessing breastfeeding practice Geneva: World Health Organization; 1991.

19. 19. Scott J, Landers M, Hughes R, Binns C. Factors associated with breastfeeding at discharge and duration of breastfeeding. Journal of Paediatric and Child Health 2001;37:254-61.

20. 20. Goyle A, Jain $\mathbf{P}$, Vyas $\mathbf{S}$, Saraf $\mathbf{H}$, Shekhawat $\mathbf{N}$. Colostrum and Prelacteal Feeding Practices followed by Families of Pavements and Roadside Squatter Settlements. Indian Journal of Social Medicine. 2004;35(1\&2):58-62.

21. 21. Taveras E, Li R, Grummer-Strawn L, Richardson $M$ Marshall R, Rego V, et al. Opinions and Practices of clinicians associated with continuation of exclusive breastfeeding. Pediatrics. 2004;113(283-290).

22. 22. Labarere J, Gelbert-Baudino N, Ayral A-S, Duc C, Berchotteau M, Bouchon N, et al. Efficacy of Breastfeeding Berchotteau M, Bouchon N, et al. Efficacy of Breastfeeding
Support Provided by Trained Clinicians During an Early, Routine, Preventive Visit: A Prospective, Randomized, Open Trial of 226 Mother-Infant Pairs. Pediatrics. 2005;115:139-46. 